FACTA UNIVERSITATIS (NIŠ)

Ser. Math. Inform. Vol. 35, No 5 (2020), 1369-1379

https://doi.org/10.22190/FUMI2005369E

\title{
ON DISCRETE WEIGHTED STATISTICAL CONVERGENCE
}

\author{
Sinan Ercan, Yavuz Altın and Rifat Çolak
}

(C) 2020 by University of Niš, Serbia | Creative Commons Licence: CC BY-NC-ND

\begin{abstract}
In the present paper, the notion of discrete weighted mean method of summability have been extended over the concept of statistical convergence. We have also given the notion of statistical $\left(M, P_{\lambda}\right)$ - summability and $\left[M, P_{\lambda}\right]_{q}$-summability. We have introduced some properties of these modes of convergence.

Keywords: statistical convergence; weighted statistical convergence; statistical $\left(\bar{N}, p_{n}\right)$ summability.
\end{abstract}

\section{Introduction}

Zygmund [16] introduced the idea of statistical convergence in 1935. Fast and Steinhaus introduced statistical convergence to assign limit to sequences which are not convergent in the usual sense in the same year (see [4],[14]). They used the asymptotic density of a set $A \subset \mathbb{N}$ which is defined as follows:

$$
\delta(A)=\lim _{n \rightarrow \infty} \frac{1}{n}|\{k \leq n: k \in A\}|,
$$

whenever the limit exists. $|\{\}$.$| indicates the cardinality of the enclosed set. A se-$ quence $x=\left(x_{k}\right)$ of numbers is called statistically convergent to a number $\ell$ provided that for $\varepsilon>0$,

$$
\lim _{n \rightarrow \infty} \frac{1}{n}\left|\left\{k \leq n:\left|x_{k}-\ell\right| \geq \varepsilon\right\}\right|=0 .
$$

In this case, we write $S-\lim _{k \rightarrow \infty} x_{k}=\ell . S$ indicates the set of all statistically convergent sequences. This notion is used an effective tool to resolve many problems in ergodic theory, fuzzy set theory, trigonometric series and Banach spaces. It was studied in summability theory by Kolk et al. (see [8]). Also,. many researchers

Received November 23, 2018; accepted July 22, 2019

2020 Mathematics Subject Classification. Primary 40A05; Secondary 40D25, 40G05, 40G15 
studied related topics with summability theory (see[2], [3], [5], [12], [13] ). Furthermore, another type of Cesaro summability was studied by Armitage and Maddox [1].

Moricz and Orhan [11] defined the notion of statistical $\left(\bar{N}, p_{n}\right)$ - summability as: Let $p=\left(p_{k}\right)$ be a sequence of nonnegative real numbers such that $p_{0}>0$, $P_{n}=\sum_{k=0}^{n} p_{k} \rightarrow \infty$ as $n \rightarrow \infty$ and $t_{n}=\frac{1}{P_{n}} \sum_{k=0}^{n} p_{k} x_{k}, n=0,1,2, \ldots$. The sequence $x=\left(x_{k}\right)$ is statistically summable to $\ell$ by the weighted mean method determined by the sequence $\left(p_{k}\right)$ or briefly statistically $\left(\bar{N}, p_{n}\right)$ - summable if

$$
s t-\lim _{n \rightarrow \infty} t_{n}=\ell \text {. }
$$

$(\bar{N}, s t)$ indicates the set of statistically $\left(\bar{N}, p_{n}\right)$ - summable sequences.

Weighted statistical convergence is introduced by Karakaya and Chisti in [7]. Also Küçükaslan studied this notion in [9]. Then the modified definition is given by Mursaleen et al. in [10] as follows:

A sequence $x=\left(x_{k}\right)$ is weighted statistically convergent (or $S_{\bar{N}^{-} \text {-convergent) to }}$ $\ell$ if for every $\varepsilon>0$, the set $\left\{k \in \mathbb{N}: p_{k}\left|x_{k}-\ell\right| \geq \varepsilon\right\}$ has weighted density zero, i.e.

$$
\lim _{n \rightarrow \infty} \frac{1}{P_{n}}\left|\left\{k \leq P_{n}: p_{k}\left|x_{k}-\ell\right| \geq \varepsilon\right\}\right|=0,
$$

where $\mathbb{N}=\{1,2, \ldots\}$. This limit is indicated by $S_{\bar{N}}-\lim _{k \rightarrow \infty} x_{k}=\ell . S(\bar{N})$ denotes the set of these kind of sequences.

The concept of weighted statistical convergence of order $\alpha$ is studied by Ghosal in [6]. Watson introduce the notion of discrete weighted mean method of summability in [15] as follows:

A sequence $\left(x_{k}\right)$ is limitable to $\ell$ by the discrete weighted mean method, if

$$
\tau_{n}=t_{\left[\lambda_{n}\right]}=\frac{1}{P_{\left[\lambda_{n}\right]}} \sum_{k=0}^{\left[\lambda_{n}\right]} p_{k} x_{k} \rightarrow \ell
$$

as $n \rightarrow \infty$ where $\left(\lambda_{n}\right)$ is a real sequence satisfies $1 \leq \lambda_{0}<\lambda_{1}<\ldots \rightarrow \infty$ and $P_{\left[\lambda_{n}\right]}=\sum_{k=0}^{\left[\lambda_{n}\right]} p_{k} \rightarrow \infty$ as $n \rightarrow \infty$ for $p_{0}>0$ and $\left[\lambda_{n}\right]$ denotes the integer part of the number $\lambda_{n}$. The set of these kind sequences denoted by $\left(M_{P_{\lambda}}\right)$.

\section{Main Results}


In this part, first we give the concepts of discrete weighted statistical convergence, $\left[M, P_{\lambda}\right]_{q^{-}}$summability and statistical $\left(M, P_{\lambda}\right)$-summability. Then we establish the relationship between these concepts. The discrete weighted density of a set $K \subseteq \mathbb{N}$ is defined by

$$
\delta_{M}(K)=\lim _{n \rightarrow \infty} \frac{1}{P_{\left[\lambda_{n}\right]}}\left|\left\{k \leq P_{\left[\lambda_{n}\right]}: k \in K\right\}\right| .
$$

In particular, if we choose $\lambda_{n}=n$ and $p_{n}=1$, then the discrete weighted density reduces to the natural density.

Throughout this paper $\left(p_{k}\right)$, is a sequence of nonnegative real numbers with $p_{1}>0$ and $P_{\left[\lambda_{n}\right]}=\sum_{k=1}^{\left[\lambda_{n}\right]} p_{k} \rightarrow \infty$ as $n \rightarrow \infty$ and $\lambda=\left(\lambda_{n}\right)$ is a real sequence satisfies $1 \leq \lambda_{1}<\lambda_{2}<\ldots \rightarrow \infty$ as $n \rightarrow \infty$ and we use the notations $\Lambda, \widehat{P}, E\left(\lambda_{n}\right), E(\lambda)$ such as

$$
\begin{gathered}
\Lambda=\left\{\lambda=\left(\lambda_{n}\right): 1 \leq \lambda_{1}<\lambda_{2}<\ldots \rightarrow \infty \text { as } n \rightarrow \infty\right\}, \\
\widehat{P}=\left\{p=\left(p_{k}\right): p_{1}>0, p_{k} \geq 0, k=2,3, \ldots \text { and } P_{\left[\lambda_{n}\right]}=\sum_{k=1}^{\left[\lambda_{n}\right]} p_{k} \rightarrow \infty \text { as } n \rightarrow \infty\right\}, \\
E\left(\lambda_{n}\right)=\left\{k \leq\left[\lambda_{n}\right]: k \in \mathbb{N}\right\}
\end{gathered}
$$

and

$$
E(\lambda)=\left\{\left[\lambda_{n}\right]: n \in \mathbb{N}\right\}=\left\{\left[\lambda_{1}\right],\left[\lambda_{2}\right],\left[\lambda_{3}\right], \ldots\right\}
$$

for a sequence $\lambda=\left(\lambda_{n}\right) \in \Lambda$. Also $A \Delta B=(A \backslash B) \cup(B \backslash A)$.

Definition 2.1 Let $\lambda=\left(\lambda_{n}\right) \in \Lambda$ and $p=\left(p_{k}\right) \in \widehat{P}$ be given. A sequence $x=\left(x_{k}\right)$ is said to be discrete weighted statistically convergent (briefly $S\left(M_{P_{\lambda}}\right)$ convergent) to $\ell$ if the set $\left\{k \in \mathbb{N}: p_{k}\left|x_{k}-\ell\right| \geq \varepsilon\right\}$ has discrete weighted density zero for every $\varepsilon>0$, i.e.

$$
\lim _{n \rightarrow \infty} \frac{1}{P_{\left[\lambda_{n}\right]}}\left|\left\{k \leq P_{\left[\lambda_{n}\right]}: p_{k}\left|x_{k}-\ell\right| \geq \varepsilon\right\}\right|=0 .
$$

It is indicated by $S\left(M_{P_{\lambda}}\right)-\lim _{k \rightarrow \infty} x_{k}=\ell . S\left(M_{P_{\lambda}}\right)$ indicates the set of these kind of sequences.

Definition 2.2 Let $\lambda=\left(\lambda_{n}\right) \in \Lambda$ and $p=\left(p_{k}\right) \in \widehat{P}$ be given. A sequence $x=\left(x_{k}\right)$ is called $\left[M, P_{\lambda}\right]_{q}$-summable $(0<q<\infty)$ to the limit $\ell$ if

$$
\lim _{n \rightarrow \infty} \frac{1}{P_{\left[\lambda_{n}\right]}} \sum_{k=1}^{\left[\lambda_{n}\right]} p_{k}\left|x_{k}-\ell\right|^{q}=0 .
$$


$\ell$ is said to be $\left[M, P_{\lambda}\right]_{q}$-limit of $x . \quad\left[M, P_{\lambda}\right]_{q}$ indicates the set of these kind of sequences.

Definition 2.3 Let $\lambda=\left(\lambda_{n}\right) \in \Lambda$ and $p=\left(p_{k}\right) \in \widehat{P}$ be given. A sequence $x=\left(x_{k}\right)$ is said to be statistically summable to $\ell$ by the discrete weighted mean method or briefly statistically $\left(M, P_{\lambda}\right)$-summable if

$$
s t-\lim _{n \rightarrow \infty} \tau_{n}=\ell \text {. }
$$

It is indicated by $\left(M, P_{\lambda}\right)-\lim _{k \rightarrow \infty} x_{k}=\ell$ and we denote by $\left(M, P_{\lambda}\right)$ the set of such sequences.

Note that for any $\left(\lambda_{n}\right)=(n+r) \in \Lambda$, where $0 \leq r<1$ is a fixed number.

(i) $\left[M, P_{\lambda}\right]_{q}$-summable reduces to $\left[\bar{N}, p_{n}\right]_{q}$-summable which is given in [10],

(ii) Discrete weighted statistical convergence is reduced to weighted statistical convergence which is given in [10],

(iii) Statistical $\left(M, P_{\lambda}\right)$-summability is reduced to statistical $\left(\bar{N}, p_{n}\right)$ - summability which is given in [11].

As a result of $(i),(i i)$ and $(i i i)$ we have that $\left[M, P_{\lambda}\right]_{q}$ includes $\left[\bar{N}, p_{n}\right]_{q}, S\left(M_{P_{\lambda}}\right)$ includes $S(\bar{N})$ and $\left(M, P_{\lambda}\right)$ includes $\bar{N}(s t)$, respectively.

We first begin with the following property. In the proof of the following Theorem we use the technique used by Watson in [15].

Theorem 2.4 Let $\lambda=\left(\lambda_{n}\right), \mu=\left(\mu_{n}\right) \in \Lambda$ be given. Then

(i) $\left[M, P_{\lambda}\right]_{q} \subseteq\left[M, P_{\mu}\right]_{q}$ if $E(\mu) \backslash E(\lambda)$ is finite,

(ii) If $p_{k}>0$ for each $k$ and if $\left[M, P_{\lambda}\right]_{q} \subseteq\left[M, P_{\mu}\right]_{q}$ holds, then $E(\mu) \backslash E(\lambda)$ is finite.

Proof. (i) Assume that $E(\mu) \backslash E(\lambda)$ is finite. Then we have an integer $n_{0}$ such that $\left\{\left[\mu_{n}\right]: n \geq n_{0}\right\} \subseteq E(\lambda)$. That is, there is an increasing sequence $\left(j_{n}\right)$ of positive integers such that $j_{n} \rightarrow \infty$ and $\left[\mu_{n}\right]=\left[\lambda_{j_{n}}\right]$ for $n \geq n_{0}$. If a sequence $x=\left(x_{n}\right)$ is statistically $\left[M, P_{\lambda}\right]_{q}-$ summable to $\ell$, then we have

$$
\frac{1}{P_{\left[\mu_{n}\right]}} \sum_{k=1}^{\left[\mu_{n}\right]} p_{k}\left|x_{k}-\ell\right|^{q}=\frac{1}{P_{\left[\lambda_{j_{n}}\right]}} \sum_{k=1}^{\left[\lambda_{j_{n}}\right]} p_{k}\left|x_{k}-\ell\right|^{q}
$$

for $n \geq n_{0}$, which gives that $x=\left(x_{n}\right)$ is statistically $\left[M, P_{\mu}\right]_{q}$-summable to $\ell$ $(0<q<\infty)$.

(ii) Suppose that $\left[M, P_{\lambda}\right]_{q} \subseteq\left[M, P_{\mu}\right]_{q}$ but $E(\mu) \backslash E(\lambda)$ is infinite. Then there is a strictly increasing sequence $\left(\left[\mu_{n_{j}}\right]\right)$ such that $\left[\mu_{n_{j}}\right] \notin E(\lambda)$, for $j=1,2,3, \ldots$. 
Consider that $\tau_{n}=t_{\left[\lambda_{n}\right]}=\frac{1}{P_{\left[\lambda_{n}\right]}} \sum_{k=1}^{\left[\lambda_{n}\right]} p_{k} x_{k}$. Then define a sequence $\left(\tau_{n}\right)$ as follows.

$$
\tau_{n}=\left\{\begin{array}{ccc}
0 & \text { if } & {\left[\lambda_{n}\right] \neq\left[\mu_{n_{j}}\right]} \\
(-1)^{j} & \text { if } & {\left[\lambda_{n}\right]=\left[\mu_{n_{j}}\right]}
\end{array}\right.
$$

Using the equality $P_{\left[\lambda_{n}\right]} t_{\left[\lambda_{n}\right]}-P_{\left[\lambda_{n}\right]-1} t_{\left[\lambda_{n}\right]-1}=P_{\left[\lambda_{n}\right]} x_{\left[\lambda_{n}\right]}$, we have $x=\left(x_{n}\right) \in$ $\left[M, P_{\lambda}\right]_{q}$ since $t_{\left[\lambda_{n}\right]}=0$ for all $n$. But the sequence is not in $\left[M, P_{\mu}\right]_{q}$.

We have the below results from Theorem 2.4.

Corollary 2.5 Let $\lambda=\left(\lambda_{n}\right), \mu=\left(\mu_{n}\right) \in \Lambda$ be given and assume that $p_{k}>0$ for every $k$. Then

(i) $\left[M, P_{\lambda}\right]_{q} \subseteq\left[M, P_{\mu}\right]_{q}$ if and only if $E(\mu) \backslash E(\lambda)$ is a finite set.

(ii) $\left[M, P_{\lambda}\right]_{q}=\left[M, P_{\mu}\right]_{q}$ if and only if $E(\lambda) \Delta E(\mu)$ is finite set.

Corollary 2.6 Let $\lambda=\left(\lambda_{n}\right), \mu=\left(\mu_{n}\right) \in \Lambda$ be given and assume that $p_{k}>0$ for every $k$. Then

(i) $\left(M, P_{\lambda}\right) \subseteq\left(M, P_{\mu}\right)$ if and only if $E(\mu) \backslash E(\lambda)$ is a finite set.

(ii) $\left(M, P_{\lambda}\right)=\left(M, P_{\mu}\right)$ if and only if $E(\lambda) \Delta E(\mu)$ is a finite set.

The following property has been given formerl, but it is also seen clearly from Theorem 2.4 by taking $\lambda=\left(\lambda_{n}\right)=(n)$.

Corollary 2.7 For any $\left(\mu_{n}\right) \in \Lambda$ the inclusion $\left[\bar{N}, p_{n}\right]_{q} \subseteq\left[M, P_{\mu}\right]_{q}$ is satisfied, where $0<q<\infty$.

Corollary 2.8 For any $\left(\lambda_{n}\right) \in \Lambda\left[M, P_{\lambda}\right]_{q} \subseteq\left[\bar{N}, p_{n}\right]_{q}$ is satisfied if $\{1,2,3, \ldots\} \backslash E(\lambda)$ is finite.

The following results can be obtained from Corollary 2.5 and Corollary 2.6.

Corollary $2.9(i)\left(\bar{N}, p_{n}\right) \subseteq\left(M, P_{\mu}\right)$ for any $\mu=\left(\mu_{n}\right) \in \Lambda$.

(ii) $\left(M, P_{\lambda}\right) \subseteq\left(\bar{N}, p_{n}\right)$ if $\{1,2,3, \ldots\} \backslash E(\lambda)$ is a finite set.

Theorem 2.10 If a sequence $x=\left(x_{k}\right)$ is $\left(M_{P_{\lambda}}\right)$-summable to $\ell$, then it is $S\left(M_{P_{\lambda}}\right)$-convergent to $\ell$. The inverse implication need not be true.

Proof. Let $x \in\left(M_{P_{\lambda}}\right)$ and define the set $K_{P_{\lambda}}(\varepsilon)=\left\{k \leq P_{\left[\lambda_{n}\right]}: p_{k}\left|x_{k}-\ell\right| \geq \varepsilon\right\}$ for $\varepsilon>0$. Hence the inequality which we have

$$
\frac{1}{P_{\left[\lambda_{n}\right]}} \sum_{k=1}^{\left[\lambda_{n}\right]} p_{k}\left|x_{k}-\ell\right|=\frac{1}{P_{\left[\lambda_{n}\right]}}\left(\sum_{k \in K_{P_{\lambda}}(\varepsilon)}+\sum_{k \notin K_{P_{\lambda}}(\varepsilon)}\right) p_{k}\left|x_{k}-\ell\right|
$$




$$
\begin{aligned}
& \geq \frac{1}{P_{\left[\lambda_{n}\right]}} \sum_{k \in K_{P_{\lambda}}(\varepsilon)} p_{k}\left|x_{k}-\ell\right| \\
& \geq \frac{1}{P_{\left[\lambda_{n}\right]}} \sum_{k \in K_{P_{\lambda}}(\varepsilon)} \varepsilon \\
& =\varepsilon \cdot \frac{1}{P_{\left[\lambda_{n}\right]}}\left|\left\{k \leq P_{\left[\lambda_{n}\right]}: p_{k}\left|x_{k}-\ell\right| \geq \varepsilon\right\}\right| .
\end{aligned}
$$

This implies that $x \in S\left(M_{P_{\lambda}}\right)$. To see the converse implication is not true, consider $\lambda_{n}=n, p_{k}=1$ for all $k$ and define $x=\left(x_{k}\right)$ by

$$
x_{k}=\left\{\begin{array}{cc}
m^{3}, & k=m^{2} \\
0, & k \neq m^{2}
\end{array} \quad m=1,2, \ldots .\right.
$$

Now we have

$$
\lim _{n \rightarrow \infty} \frac{1}{n}\left|\left\{k \leq n: 1 .\left|x_{k}-0\right| \geq \varepsilon\right\}\right|=0 \text { and } \lim _{n \rightarrow \infty} \frac{1}{n} \sum_{k=1}^{n} 1\left|x_{k}-0\right|=\infty .
$$

This means $x \in S\left(M_{P_{\lambda}}\right)$ but $x \notin\left(M_{P_{\lambda}}\right)$.

Theorem 2.11 Assume that $p_{n} \geq 1$ for all $n$ and

$$
1 \leq \lim _{n \rightarrow \infty} \frac{P_{\left[\lambda_{n}\right]}}{n}<\infty
$$

holds . Then $x \in S$ if $x \in S\left(M_{P_{\lambda}}\right)$. The opposite case is not true.

Proof. Suppose that $p_{n} \geq 1$ and (2.1) holds for all $n \in \mathbb{N}$. Let $x \in S\left(M_{P_{\lambda}}\right)$, then we have

$$
\begin{aligned}
\frac{1}{n}\left|\left\{k \leq n:\left|x_{k}-\ell\right| \geq \varepsilon\right\}\right| & \leq \frac{1}{n}\left|\left\{k \leq n: p_{k}\left|x_{k}-\ell\right| \geq \varepsilon\right\}\right| \\
& \leq \frac{P_{\left[\lambda_{n}\right]}}{n} \frac{1}{P_{\left[\lambda_{n}\right]}}\left|\left\{k \leq P_{\left[\lambda_{n}\right]}: p_{k}\left|x_{k}-\ell\right| \geq \varepsilon\right\}\right|
\end{aligned}
$$

for $\varepsilon>0$. As $n \rightarrow \infty$ we obtain $x \in S$.

To see the opposite case is not true, consider the sequence $x=\left(x_{k}\right)$ defined by

$$
x_{k}=\left\{\begin{array}{cc}
1 & \text { if } k=m^{2} \\
\frac{1}{\sqrt{k}} & \text { if } k \neq m^{2}
\end{array} \quad m \in \mathbb{N} .\right.
$$

The sequence $x=\left(x_{k}\right)$ is a statistically convergent to 0 , but it is not discrete weighted statistical convergent to 0 , while $p_{k}=k$ for $k \in \mathbb{N}$.

The sufficient condition to be true the converse implication is given the following theorem. 
Theorem 2.12 Assume that $p_{n}<1$ and

$$
1 \leq \lim _{n \rightarrow \infty} \frac{n}{P_{\left[\lambda_{n}\right]}}<\infty
$$

for all $n \in \mathbb{N}$. If $x \in S$, then $x \in S\left(M_{P_{\lambda}}\right)$.

Proof. Suppose that $p_{n}<1$ and (2.2) holds for all $n \in \mathbb{N}$. Let $x \in S$ then we have

$$
\begin{aligned}
\frac{1}{P_{\left[\lambda_{n}\right]}}\left|\left\{k \leq P_{\left[\lambda_{n}\right]}: p_{k}\left|x_{k}-\ell\right| \geq \varepsilon\right\}\right| & \leq \frac{1}{P_{\left[\lambda_{n}\right]}}\left|\left\{k \leq P_{\left[\lambda_{n}\right]}:\left|x_{k}-\ell\right| \geq \varepsilon\right\}\right| \\
& \leq \frac{n}{P_{\left[\lambda_{n}\right]}} \frac{1}{n}\left|\left\{k \leq n:\left|x_{k}-\ell\right| \geq \varepsilon\right\}\right|
\end{aligned}
$$

for any $\varepsilon>0$. Hence we obtain the desired result as $n \rightarrow \infty$.

Theorem 2.13 $S\left(M_{P_{\lambda}}\right)$-lim of an $S\left(M_{P_{\lambda}}\right)$-convergent sequence is unique.

Proof. Suppose the sequence $x=\left(x_{k}\right)$ is $S\left(M_{P_{\lambda}}\right)$-convergent both to $\ell_{1}$ and $\ell_{2}$. If possible let $\ell_{1} \neq \ell_{2}$ and choose $\varepsilon=\frac{1}{2}\left|\ell_{1}-\ell_{2}\right|>0$ and $p_{k}>c>0$ for all $k$. Then

$$
\begin{aligned}
1 & \leq \frac{1}{P_{\left[\lambda_{n}\right]}}\left|\left\{k \leq P_{\left[\lambda_{n}\right]}: p_{k}\left|\ell_{1}-\ell_{2}\right| \geq \varepsilon c\right\}\right| \\
& \leq \frac{1}{P_{\left[\lambda_{n}\right]}}\left|\left\{k \leq P_{\left[\lambda_{n}\right]}: p_{k}\left|x_{k}-\ell_{1}\right| \geq \frac{\varepsilon c}{2}\right\}\right|+\frac{1}{P_{\left[\lambda_{n}\right]}}\left|\left\{k \leq P_{\left[\lambda_{n}\right]}: p_{k}\left|x_{k}-\ell_{2}\right| \geq \frac{\varepsilon c}{2}\right\}\right| .
\end{aligned}
$$

This is impossible because right hand side tends to 0 as $n \rightarrow \infty$. Hence we have desired result $\ell_{1}=\ell_{2}$.

Theorem 2.14 Let the sequence $\left(p_{k}\left|\left(x_{k}-\ell\right)\right|\right)$ be bounded. Then $x=\left(x_{k}\right)$ is statistically $\left(M_{P_{\lambda}}\right)$ - summable to $\ell$ if it is $S\left(M_{P_{\lambda}}\right)$-convergent to $\ell$, but the opposite case is not true.

Proof. Suppose $p_{k}\left|\left(x_{k}-\ell\right)\right| \leq T$ for every $k$, for some constant $T$ and assume that the sequence $x=\left(x_{k}\right)$ is $S\left(M_{P_{\lambda}}\right)$-convergent to $\ell$. We have

$$
\begin{aligned}
\left|\tau_{n}-\ell\right| & =\left|\frac{1}{P_{\left[\lambda_{n}\right]}} \sum_{k=1}^{\left[\lambda_{n}\right]} p_{k} x_{k}-\ell\right| \\
& =\left|\frac{1}{P_{\left[\lambda_{n}\right]}} \sum_{k=1}^{\left[\lambda_{n}\right]} p_{k}\left(x_{k}-\ell\right)\right|
\end{aligned}
$$

Theorem 2.15 Let the sequence $\left(p_{k}\left|\left(x_{k}-\ell\right)\right|\right)$ be bounded. Then $x=\left(x_{k}\right)$ is statistically $\left(M_{P_{\lambda}}\right)$ - summable to $\ell$ if it is $S\left(M_{P_{\lambda}}\right)$-convergent to $\ell$, but the opposite case is not true. 
Proof. Suppose $p_{k}\left|\left(x_{k}-\ell\right)\right| \leq T$ for every $k$, for some constant $T$ and assume that the sequence $x=\left(x_{k}\right)$ is $S\left(M_{P_{\lambda}}\right)$-convergent to $\ell$. We have

$$
\begin{aligned}
& \begin{aligned}
\left|\tau_{n}-\ell\right| & =\left|\frac{1}{P_{\left[\lambda_{n}\right]}} \sum_{k=1}^{\left[\lambda_{n}\right]} p_{k} x_{k}-\ell\right| \\
& =\left|\begin{array}{c}
\frac{1}{P_{\left[\lambda_{n}\right]}} \sum_{k=1}^{\left[\lambda_{n}\right]} p_{k}\left(x_{k}-\ell\right)
\end{array}\right|
\end{aligned} \\
& =\left|\frac{1}{P_{\left[\lambda_{n}\right]}} \sum_{\substack{k=1 \\
k \in K_{P_{\lambda}}(\varepsilon)}}^{\left[\lambda_{n}\right]} p_{k}\left(x_{k}-\ell\right)+\frac{1}{P_{\left[\lambda_{n}\right]}} \sum_{\substack{k=1 \\
k \in K_{P_{\lambda}}^{c}(\varepsilon)}}^{\left[\lambda_{n}\right]} p_{k}\left(x_{k}-\ell\right)\right| \\
& \leq\left|\frac{1}{P_{\left[\lambda_{n}\right]}} \sum_{\substack{k=1 \\
k \in K_{P_{\lambda}}(\varepsilon)}}^{\left[\lambda_{n}\right]} p_{k}\left(x_{k}-\ell\right)\right|+\left|\frac{1}{P_{\left[\lambda_{n}\right]}} \sum_{\substack{k=1 \\
k \in K_{P_{\lambda}}^{c}(\varepsilon)}}^{\left[\lambda_{n}\right]} p_{k}\left(x_{k}-\ell\right)\right| \\
& \leq \frac{1}{P_{\left[\lambda_{n}\right]}} \sum_{\substack{k=1 \\
k \in K_{P_{\lambda}}(\varepsilon)}}^{\left[\lambda_{n}\right]} p_{k}\left|\left(x_{k}-\ell\right)\right|+\frac{1}{P_{\left[\lambda_{n}\right]}} \sum_{\substack{k=1 \\
k \in K_{P_{\lambda}}^{c}(\varepsilon)}}^{\left[\lambda_{n}\right]} p_{k}\left|\left(x_{k}-\ell\right)\right| \\
& \leq \frac{1}{P_{\left[\lambda_{n}\right]}} \cdot T \cdot\left|K_{P_{\lambda}}(\varepsilon)\right|+\frac{1}{P_{\left[\lambda_{n}\right]}} \sum_{k \in K_{P_{\lambda}}^{c}} \varepsilon \\
& =\frac{1}{P_{\left[\lambda_{n}\right]}} \cdot T \cdot\left|K_{P_{\lambda}}(\varepsilon)\right|+\varepsilon \frac{\left|K_{P_{\lambda}}^{c}(\varepsilon)\right|}{P_{\left[\lambda_{n}\right]}} \rightarrow 0
\end{aligned}
$$

as $n \rightarrow \infty$, where $K_{P_{\lambda}}(\varepsilon)=\left\{k \leq P_{\left[\lambda_{n}\right]}: p_{k}\left|x_{k}-\ell\right| \geq \varepsilon\right\}$. This means that $\tau_{n} \rightarrow$ $\ell$ as $n \rightarrow \infty$. That is, $x$ is $\left(M_{P_{\lambda}}\right)$-summable to $\ell$ and hence it is statistically $\left(M_{P_{\lambda}}\right)$-summable to $\ell$.

To see that the opposite case is not true, let $p_{k}=1$ for every $k \in \mathbb{N}$. Consider the sequence $x=\left(x_{k}\right)$ defined by

$$
x_{k}=\left\{\begin{array}{cc}
1 & \text { if } k=m^{2}-m, m^{2}-m+1, \ldots, m^{2}-1 ; \\
-m & \text { if } k=m^{2} ; \\
0 & \text { otherwise. }
\end{array}\right.
$$

where $m=2,3,4, \ldots$. Then we have, for $s=0,1,2, \ldots, m-1 ; m=2,3, \ldots$

$$
\tau_{n}=t_{\left[\lambda_{n}\right]}=\frac{1}{\left[\lambda_{n}\right]+1} \sum_{k=0}^{\left[\lambda_{n}\right]} x_{k}=\left\{\begin{array}{cc}
\frac{s+1}{\left[\lambda_{n}\right]+1} & \text { if }\left[\lambda_{n}\right]=m^{2}-m+s \\
0 & \text { otherwise. }
\end{array}\right.
$$

It is easy to see that $\lim _{n \rightarrow \infty} \tau_{n}=0$ and hence $s t-\lim _{n \rightarrow \infty} \tau_{n}=0$, i.e. $x=\left(x_{k}\right)$ is statistically $\left(M_{P_{\lambda}}\right)$-summable to 0 . On the other hand $s t-\lim _{k \rightarrow \infty} \inf x_{k}=0$ and st $-\lim _{k \rightarrow \infty} \sup x_{k}=1$, because

$$
\delta\left(\left\{k: k=m^{2}, m=1,2,3, \ldots\right\}\right)=0,
$$




$$
\delta\left(\left\{k: k \neq m^{2}-m, m^{2}-m+1, \ldots, m^{2}-1, m ; m=2,3, \ldots\right\}\right) \neq 0
$$

and

$$
\delta\left(\left\{k: k=m^{2}-m, m^{2}-m+1, \ldots, m^{2}-1 ; m=2,3, \ldots\right\}\right) \neq 0 .
$$

Hence $x=\left(x_{k}\right)$ is not $S\left(M_{P_{\lambda}}\right)$-convergent.

Theorem 2.16 Let a sequence $x=\left(x_{k}\right)$ be $\left[M_{P_{\lambda}}\right]_{q}$-summable to $\ell$. If $0<q<1$ and $0 \leq\left|x_{k}-\ell\right|<1$ or $1 \leq q<\infty$ and $1 \leq\left|x_{k}-\ell\right|<\infty$, then $x=\left(x_{k}\right)$ is $S\left(M_{P_{\lambda}}\right)$-statistically convergent to $\ell$.

Proof. We have $p_{k}\left|x_{k}-\ell\right|^{q} \geq p_{k}\left|x_{k}-\ell\right|$ for both cases. Then

$$
\begin{aligned}
\frac{1}{P_{\left[\lambda_{n}\right]}} \sum_{k=1}^{\left[\lambda_{n}\right]} p_{k}\left|x_{k}-\ell\right|^{q} & \geq \frac{1}{P_{\left[\lambda_{n}\right]}} \sum_{k=1}^{\left[\lambda_{n}\right]} p_{k}\left|x_{k}-\ell\right| \\
& \geq \frac{1}{P_{\left[\lambda_{n}\right]}} \sum_{\substack{k=1 \\
k \in K_{P_{\lambda}}(\varepsilon)}}^{\left[\lambda_{n}\right]} p_{k}\left|x_{k}-\ell\right| \\
& \geq \frac{1}{P_{\left[\lambda_{n}\right]}} \sum_{\substack{k=1 \\
k \in K_{P_{\lambda}}(\varepsilon)}}^{\left[\lambda_{n}\right]} \\
& =\varepsilon \frac{\left|K_{P_{\lambda}}(\varepsilon)\right|}{P_{\left[\lambda_{n}\right]}} .
\end{aligned}
$$

Since $\frac{1}{P_{\left[\lambda_{n}\right]}} \sum_{k=1}^{\left[\lambda_{n}\right]} p_{k}\left|x_{k}-\ell\right|^{q} \rightarrow 0$ as $n \rightarrow \infty$ we have $\lim _{n \rightarrow \infty} \frac{1}{P_{\left[\lambda_{n}\right]}}\left|K_{P_{\lambda}}(\varepsilon)\right|=0$. This means that $x=\left(x_{k}\right)$ is $S\left(M_{P_{\lambda}}\right)$-convergent to $\ell$.

Theorem 2.17 Let the sequence $\left(p_{k}\left|\left(x_{k}-\ell\right)\right|\right)$ be bounded and let a sequence $x=\left(x_{k}\right)$ be $S\left(M_{P_{\lambda}}\right)$-convergent to $\ell$. If $0<q<1$ and $0 \leq T<\infty$ or $1 \leq q<\infty$ and $0 \leq T<1$, then $x=\left(x_{k}\right)$ is $\left[M_{P_{\lambda}}\right]_{q}$-summable to $\ell$.

Proof. Assume that $x=\left(x_{k}\right)$ is $S\left(M_{P_{\lambda}}\right)$-convergent to $\ell$. Since $p_{k}\left|x_{k}-\ell\right| \leq T$ $(k=1,2, \ldots)$ for some $T \geq 0$, we have

$$
\begin{aligned}
\frac{1}{P_{\left[\lambda_{n}\right]}} \sum_{k=1}^{\left[\lambda_{n}\right]} p_{k}\left|x_{k}-\ell\right|^{q} & =\frac{1}{P_{\left[\lambda_{n}\right]}} \sum_{\substack{k=1 \\
k \notin K_{P_{\lambda}}(\varepsilon)}}^{\left[\lambda_{n}\right]} p_{k}\left|x_{k}-\ell\right|^{q}+\frac{1}{P_{\left[\lambda_{n}\right]}} \sum_{\substack{k=1 \\
k \in K_{P_{\lambda}}(\varepsilon)}}^{\left[\lambda_{n}\right]} p_{k}\left|x_{k}-\ell\right|^{q} \\
& =s_{1}\left(\left[\lambda_{n}\right]\right)+s_{2}\left(\left[\lambda_{n}\right]\right)
\end{aligned}
$$

where

$$
s_{1}\left(\left[\lambda_{n}\right]\right)=\frac{1}{P_{\left[\lambda_{n}\right]}} \sum_{\substack{k=1 \\ k \notin K_{P_{\lambda}}(\varepsilon)}}^{\left[\lambda_{n}\right]} p_{k}\left|x_{k}-\ell\right|^{q}
$$


and

$$
s_{2}\left(\left[\lambda_{n}\right]\right)=\frac{1}{P_{\left[\lambda_{n}\right]}} \sum_{\substack{k=1 \\ k \in K_{P_{\lambda}}(\varepsilon)}}^{\left[\lambda_{n}\right]} p_{k}\left|x_{k}-\ell\right|^{q} .
$$

Now we have

$$
\begin{aligned}
s_{1}\left(\left[\lambda_{n}\right]\right) & =\frac{1}{P_{\left[\lambda_{n}\right]}} \sum_{\substack{k=1 \\
k \notin K_{P_{\lambda}}(\varepsilon)}}^{\left[\lambda_{n}\right]} p_{k}\left|x_{k}-\ell\right|^{q} \\
& \leq \frac{1}{P_{\left[\lambda_{n}\right]}} \sum_{\substack{k=1 \\
k \notin \lambda_{P_{\lambda}}(\varepsilon)}}^{\left[\lambda_{n}\right]} p_{k}\left|x_{k}-\ell\right|=\varepsilon \frac{1}{P_{\left[\lambda_{n}\right]}}\left|K_{P_{\lambda}}^{c}(\varepsilon)\right| \rightarrow 0
\end{aligned}
$$

and

$$
\begin{aligned}
s_{2}\left(\left[\lambda_{n}\right]\right) & =\frac{1}{P_{\left[\lambda_{n}\right]}} \sum_{\substack{k=1 \\
k \in K_{P_{\lambda}}(\varepsilon)}}^{\left[\lambda_{n}\right]} p_{k}\left|x_{k}-\ell\right|^{q} \leq \frac{1}{P_{\left[\lambda_{n}\right]}} \sum_{\substack{k=1 \\
k \in K_{P_{\lambda}}(\varepsilon)}}^{\left[\lambda_{n}\right]} p_{k}\left|x_{k}-\ell\right| \\
& \leq\left(\sup _{k} p_{k}\left|x_{k}-\ell\right|\right)\left(\left|K_{P_{\lambda}}(\varepsilon)\right| / P_{\left[\lambda_{n}\right]}\right) \leq T\left|K_{P_{\lambda}}(\varepsilon)\right| / P_{\left[\lambda_{n}\right]} \rightarrow 0
\end{aligned}
$$

as $n \rightarrow \infty$. Hence $\left(x_{k}\right)$ is $\left[M_{P_{\lambda}}\right]_{q}$-summable to $\ell$.

\section{REF E R E N C E S}

1. D. H. Armitage, I. J.Maddox: A new type of Cesàro mean. Analysis, 9(1-2) (1989), 195-206.

2. B.T. Bilalov, S. R., Sadigova: On $\mu$-statistical convergence. Proc. Amer. Math. Soc. 143(9) (2015), 3869-3878.

3. J. Connor: The statistical and strong p-Cesáro convergence of sequences. Analysis, 8 (1988), 47-63.

4. H. Fast: Sur la convergence statistique. Colloquium Math. 2 (1951), 241-244.

5. J.A. Fridy: On statistical convergence. Analysis, 5 (1985), 301-313.

6. S. Ghosal: Weighted statistical convergence of order $\alpha$ and its applications. J. Egyptian Math. Soc. 24(1) (2016), 60-67.

7. V. Karakaya, T. A. Chishti: Weighted statistical convergence. Iran. J. Sci. Technol. Trans. A Sci. 33 (2009), 219-223.

8. E. Kolk: The statistical convergence in Banach spaces. Acta et Comment. Univ. Tartu. 928 (1991), 41-52.

9. M. Küçükaslan: Weighted statistical convergence. International Journal of Science and Technology, 2 (2012), 2-10.

10. M. Mursaleen, V. Karakaya, M. Ertürk, F. Gürsoy: Weighted statistical convergence and its application to Korovkin type aprroximation theorem. Appl. Math. Comput. 218 (2012), 9132-9137. 
11. F. Moricz, C. Orhan: Tauberian conditions under which statistical convergence follows from statistical summability by weighted means. Studia Sci. Math. Hungar. 41 (2004), 391-403.

12. D. Rath, B. C. Tripathy: On statistically convergent and statistically convergent and statistically Cauchy sequences. Indian J. Pure. Appl. Math. 25(4) (1994), 381-386.

13. T. Šalăt: On statistically convergent sequences of real numbers. Math. Slovaca, 30 (1980), 139-150.

14. H. Steinhaus: Sur la convergence ordinaire et la convergence asymptotique. Colloquium Math. 2 (1951), 73-74.

15. B. Watson: Discrete weighted mean methods. Indian J. Pure Appl. Math. 30(12) (1999), 1223-1227.

16. A. Zygmund: Trigonometrical Series. Monogr. Mat., Vol. 5. Warszawa-Lwow 1935.

\author{
Sinan Ercan \\ Department of Mathematics, \\ Firat University Elazig, Türkiye \\ sinanercan45@gmail.com \\ Yavuz Altın \\ Department of Mathematics, \\ Firat University, Elazig, Türkiye \\ yaltin230yahoo.com \\ Rifat Çolak \\ Department of Mathematics \\ Firat University, Elazig, Türkiye \\ rftcolak@gmail.com
}

